\title{
The invariant and helicity amplitudes in the transitions $\Lambda_{b} \rightarrow \Lambda^{*}\left(\frac{1}{2}^{ \pm}, \frac{3}{2}^{ \pm}\right)+J / \psi$
}

\author{
Mikhail Ivanov*t \\ JINR, Dubna \\ E-mail: ivanovmetheor.jinr.ru
}

\begin{abstract}
We present results for the invariant and helicity amplitudes in the transitions $\Lambda_{b} \rightarrow \Lambda^{(*)}\left(J^{P}\right)+J / \psi$ where the $\Lambda^{(*)}\left(J^{P}\right)$ are $\Lambda($ sud $)$-type ground and excited states with $J^{P}$ quantum numbers $J^{P}=$ $\frac{1}{2}^{ \pm}, \frac{3}{2}^{ \pm}$. The calculations are performed in the framework of a covariant confined quark model. This analysis is important for the identification of the hidden charm pentaquark states $P_{c}^{+}(4380)$ and $P_{c}^{+}$(4450) which were discovered by the LHCb Coll. We also discuss the possible New Physics effects in the exclusive decays $\bar{B}^{0} \rightarrow D^{(*)} \tau^{-} \bar{v}_{\tau}$. We extend the Standard Model by taking into account right-handed vector (axial), left- and right-handed (pseudo)scalar, and tensor current contributions. The $\bar{B}^{0} \rightarrow D^{(*)}$ transition form factors are calculated in the full kinematic $q^{2}$ range by employing a covariant quark model.
\end{abstract}

The European Physical Society Conference on High Energy Physics

5-12 July 2017

Venice, Italy

\footnotetext{
* Speaker.

${ }^{\dagger}$ Talk based on the work in collaboration with T. Gutsche, J. G. Körner, V. E. Lyubovitskij, V. V. Lyubushkin, P. Santorelli and C. T. Tran.
} 


\section{Introduction}

In the last few years, the semileptonic decays $\bar{B}^{0} \rightarrow D^{(*)} \tau^{-} \bar{v}_{\tau}$ have been widely discussed in the literature as candidates for testing the Standard Model (SM) and searching for possible new physics (NP) in charged-current interactions. At $B$ factories, the Belle and BABAR Collaborations have been continuously updating their measurements with better precision based on electron-positron colliders. Recently, the LHCb Collaboration has also entered the game with data taken at the LHC hadron collider. The three groups have reported measurements of the ratios in Refs. [1, 3, 2, 4, 5]. These measurements provide the average ratios

$$
\left.R(D)\right|_{\text {expt }}=0.397 \pm 0.049,\left.\quad R\left(D^{*}\right)\right|_{\text {expt }}=0.308 \pm 0.017,
$$

which exceed the SM expectations given in Refs. [6, 7]

$$
\left.R(D)\right|_{\mathrm{SM}}=0.300 \pm 0.008,\left.\quad R\left(D^{*}\right)\right|_{\mathrm{SM}}=0.252 \pm 0.003,
$$

by $1.9 \sigma$ and $3.3 \sigma$, respectively. The excess of $R\left(D^{(*)}\right)$ over SM predictions has attracted a great deal of attention in the particle physics community and has led to many theoretical studies looking for NP explanations.

In the paper [8] we included NP operators in the effective Hamiltonian and investigated their effects on physical observables of the decays $\bar{B}^{0} \rightarrow D^{(*)} \ell^{-} \bar{v}_{\ell}$. We defined a full set of form factors corresponding to $\mathrm{SM}+\mathrm{NP}$ operators and calculated them by employing the covariant confined quark model (CCQM). In the CCQM the transition form factors can be determined in the full range of momentum transfer, making the calculations straightforward without any extrapolation. This provides an opportunity to investigate NP operators in a self-consistent manner, and independently from the HQET. We first constrain the NP operators using experimental data, then analyze their effects on various observables including the ratios of branching fractions, the forward-backward asymmetries, and a set of polarization observables. We also derive the fourfold angular distribution for the cascade decay $\bar{B}^{0} \rightarrow D^{*+}\left(\rightarrow D^{0} \pi^{+}\right) \tau^{-} \bar{v}_{\tau}$ to analyze the polarization of the $D^{*}$ meson in the presence of NP by using the traditional helicity amplitudes.

Recently the LHCb Collaboration has performed an angular analysis of the decay $\Lambda_{b} \rightarrow \Lambda^{(*)}+$ $J / \psi$, where the $\Lambda_{b}$ 's are produced in $p p$ collisions at $\sqrt{s}=7 \mathrm{TeV}$ at the LHC (CERN) [9]. They reported on the measurement of the relative magnitude of the helicity amplitudes in the decay $\Lambda_{b} \rightarrow \Lambda^{(*)}+J / \psi$ by a fit to several asymmetry parameters in the cascade decay distribution $\Lambda_{b} \rightarrow$ $\Lambda\left(\rightarrow p \pi^{-}\right)+J / \psi\left(\rightarrow \ell^{+} \ell^{-}\right)$and $\Lambda_{b} \rightarrow \Lambda^{*}\left(\rightarrow p K^{-}\right)+J / \psi\left(\rightarrow \ell^{+} \ell^{-}\right)$. In the paper [10] we have performed a detailed analysis of the decay process $\Lambda_{b} \rightarrow \Lambda+J / \psi$ within the CCQM. We have worked out two variants of the threefold joint angular decay distributions in the cascade decay $\Lambda_{b} \rightarrow \Lambda\left(\rightarrow p \pi^{-}\right)+J / \psi\left(\rightarrow \ell^{+} \ell^{-}\right)$for polarized and unpolarized $\Lambda_{b}$ decays. We have further listed results on helicity amplitudes which determine the rate and the asymmetry parameters in the decay processes $\Lambda_{b} \rightarrow \Lambda\left(\rightarrow p \pi^{-}\right)+J / \psi$ and $\Lambda_{b} \rightarrow \Lambda\left(\rightarrow p \pi^{-}\right)+\psi(2 S)$.

In the paper [11] we have calculated the corresponding invariant and helicity amplitudes in the transitions $\Lambda_{b} \rightarrow \Lambda^{(*)}\left(J^{P}\right)+J / \psi$ where the $\Lambda^{(*)}\left(J^{P}\right)$ are $\Lambda$-type (sud) ground and excited states with $J^{P}$ quantum numbers $J^{P}=\frac{1}{2}^{ \pm}, \frac{3}{2}^{ \pm}$. We found that the values of the helicity amplitudes for the $\Lambda_{b} \rightarrow \Lambda^{*}\left(1520, \frac{3}{2}^{-}\right), \Lambda^{*}\left(1890, \frac{3}{2}^{+}\right)$transitions are suppressed compared with those for the transitions to the ground state $\Lambda\left(1116, \frac{1}{2}^{+}\right)$also calculated in [10] and the excited state $\Lambda^{*}\left(1405, \frac{1}{2}^{-}\right)$. 
This analysis is important for the identification of the hidden charm pentaquark states $P_{c}^{+}(4380)$ and $P_{c}^{+}(4450)$ since the cascade decay $\Lambda_{b} \rightarrow \Lambda^{*}\left(\frac{1}{2}^{-}, \frac{3}{2}^{ \pm}\right)\left(\rightarrow p K^{-}\right)+J / \psi$ involves the same final states as the decay $\Lambda_{b}^{0} \rightarrow P_{c}^{+}(\rightarrow p J / \psi)+K^{-}$.

\section{Extended Effective Hamiltonian for $B \rightarrow D^{(*)} \tau \bar{v}_{\tau}$ decay}

We extend the SM effective Hamiltonian for the quark-level transition $b \rightarrow c \tau^{-} \bar{v}_{\tau}$ by including new operators:

$$
\begin{aligned}
\mathscr{H}_{e f f} & =2 \sqrt{2} G_{F} V_{c b}\left[\left(1+V_{L}\right) \mathscr{O}_{V_{L}}+V_{R} \mathscr{O}_{V_{R}}+S_{L} \mathscr{O}_{S_{L}}+S_{R} \mathscr{O}_{S_{R}}+T_{L} \mathscr{O}_{T_{L}}\right], \\
\mathscr{O}_{V_{L}} & =\left(\bar{c} \gamma^{\mu} P_{L} b\right)\left(\bar{\tau} \gamma_{\mu} P_{L} v_{\tau}\right), \quad \mathscr{O}_{V_{R}}=\left(\bar{c} \gamma^{\mu} P_{R} b\right)\left(\bar{\tau} \gamma_{\mu} P_{L} v_{\tau}\right), \\
\mathscr{O}_{S_{L}} & =\left(\bar{c} P_{L} b\right)\left(\bar{\tau} P_{L} v_{\tau}\right), \quad \mathscr{O}_{S_{R}}=\left(\bar{c} P_{R} b\right)\left(\bar{\tau} P_{L} v_{\tau}\right), \quad \mathscr{O}_{T_{L}}=\left(\bar{c} \sigma^{\mu v} P_{L} b\right)\left(\bar{\tau} \sigma_{\mu v} P_{L} v_{\tau}\right) .
\end{aligned}
$$

Here, $\sigma_{\mu v}=i\left[\gamma_{\mu}, \gamma_{v}\right] / 2, P_{L, R}=\left(1 \mp \gamma_{5}\right) / 2$ are the left and right projection operators, and $V_{L, R}$, $S_{L, R}$, and $T_{L}$ are the complex Wilson coefficients governing the NP contributions. In the SM one has $V_{L, R}=S_{L, R}=T_{L}=0$. We assume that NP only affects leptons of the third generation.

The form factors which appear in the matrix elements including the NP operators are calculated in the framework of the CCQM. The model parameters, namely, the hadron size parameter $\Lambda$, the constituent quark masses $m_{q_{i}}$, and the universal infrared cutoff parameter $\lambda$, are determined by fitting calculated quantities of a multitude of basic processes to available experimental data or lattice simulations. It is important to note that within the SM (without any NP operators) our model calculation yields $R(D)=0.267$ and $R\left(D^{*}\right)=0.238$ [12], which are consistent with other SM predictions given in Refs. [6, 7] within $10 \%$.

In order to acquire the allowed regions for the NP Wilson coefficients, we assume that besides the SM contribution, only one of the NP operators in Eq. (2.1) is switched on at a time. We then compare the calculated ratios $R\left(D^{(*)}\right)$ with the recent experimental data. The experimental constraints are shown in Fig. 1. The vector operators $\mathscr{O}_{V_{L, R}}$ and the left scalar operator $\mathscr{O}_{S_{L}}$ are favored while there is no allowed region for the right scalar operator $\mathscr{O}_{S_{R}}$ within $2 \sigma$. Therefore we will not consider $\mathscr{O}_{S_{R}}$ in what follows. The tensor operator $\mathscr{O}_{T_{L}}$ is less favored, but it can still well explain the current experimental results. The stringent constraint on the tensor coupling mainly comes from the experimental data of $R\left(D^{*}\right)$. In each allowed region at $2 \sigma$ we find the best-fit value for each NP coupling. The best-fit couplings read

$$
V_{L}=-0.23-i 0.85, \quad V_{R}=0.03+i 0.60, \quad S_{L}=-1.80-i 0.07, \quad T_{L}=0.38+i 0.06,
$$

and are marked with an asterisk.

The allowed regions of the coupling coefficients are then used to analyze the effect of the NP operators on different physical observables [13].

\section{The decays $\Lambda_{b} \rightarrow \Lambda^{(*)}\left(\frac{1}{2}^{ \pm}, \frac{3}{2}^{ \pm}\right)+J / \psi$ : matrix element and helicity amplitudes}

The matrix element of the exclusive decay $\Lambda_{1}\left(p_{1}, \lambda_{1}\right) \rightarrow \Lambda_{2}\left(p_{2}, \lambda_{2}\right)+V\left(q, \lambda_{V}\right)$ is defined by (in the present application the vector meson label $V$ stands for the $J / \Psi$ )

$$
M\left(\Lambda_{1} \rightarrow \Lambda_{2}+V\right)=\frac{G_{F}}{\sqrt{2}} V_{c b} V_{c s}^{*} C_{\mathrm{eff}} f_{V} M_{V}\left\langle\Lambda_{2}\left|\bar{s} O_{\mu} b\right| \Lambda_{1}\right\rangle \varepsilon^{\dagger \mu}\left(\lambda_{V}\right),
$$



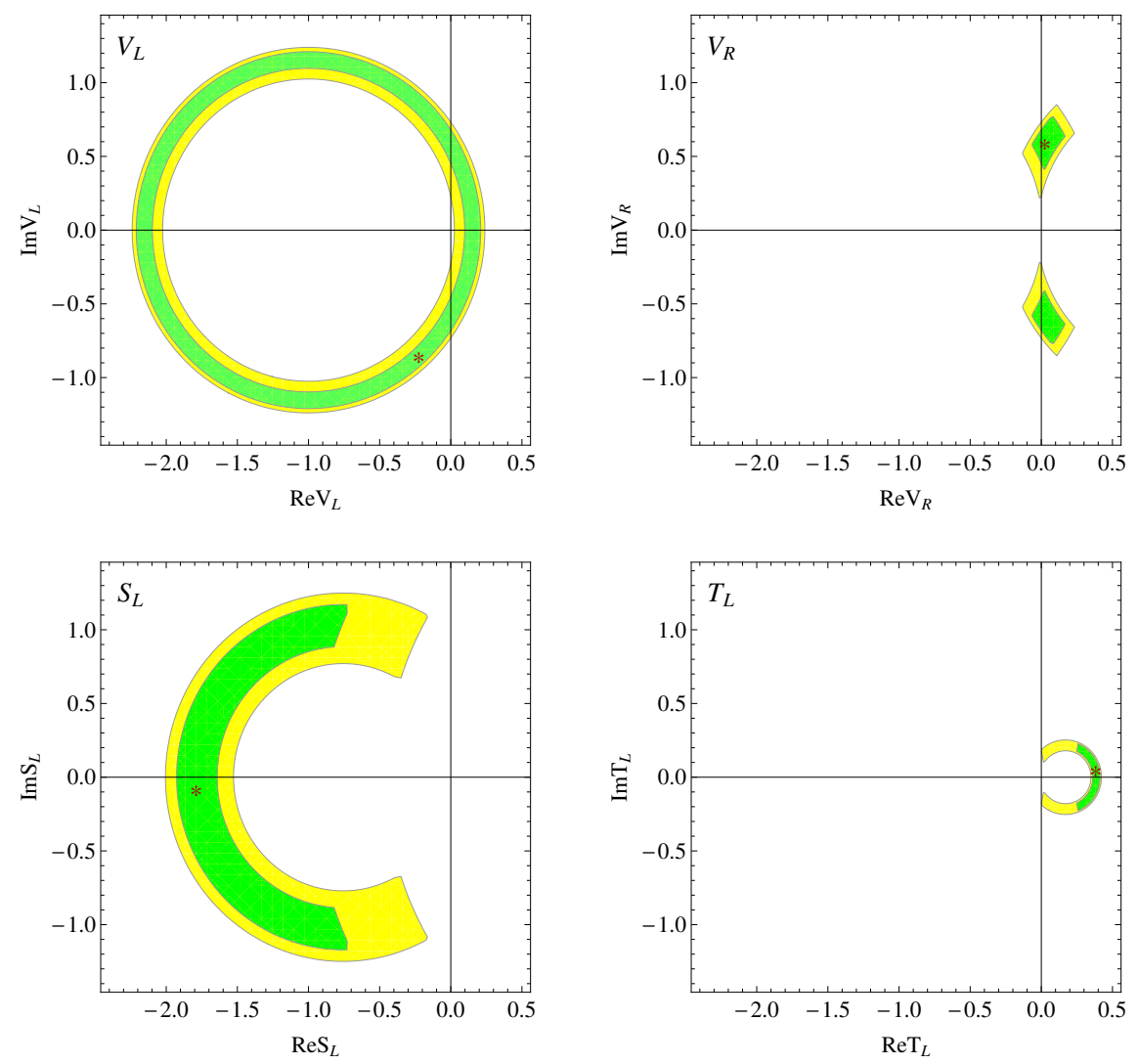

Figure 1: The allowed regions of the Wilson coefficients $V_{L, R}, S_{L}$, and $T_{L}$ within $1 \sigma$ (green, dark) and $2 \sigma$ (yellow, light). The best-fit value in each case is denoted with the symbol $*$. The coefficient $S_{R}$ is disfavored at $2 \sigma$ and therefore is not shown here.

where $M_{V}$ and $f_{V}$ are the mass and the leptonic decay constant of the vector meson $V$. The coefficient $C_{\text {eff }}$ stands for the combination of Wilson coefficients $C_{\text {eff }}=C_{1}+C_{3}+C_{5}+\xi\left(C_{2}+C_{4}+C_{6}\right)$. The color factor $\xi=1 / N_{c}$ will be set to zero such that we only keep the leading term in the $1 / N_{c}$-expansion. The hadronic matrix element $\left\langle\Lambda_{2}\left|\bar{s} O^{\mu} b\right| \Lambda_{1}\right\rangle$ is expressed in terms of six and eight, respectively, dimensionless invariant form factors $F_{i}^{V / A}\left(q^{2}\right)$.

The three-quark currents with the appropriate quantum numbers of the the $\Lambda_{Q}\left(\frac{1}{2}^{ \pm}, \frac{3}{2}^{ \pm}\right)$states are given by

$$
\begin{array}{ll}
\Lambda_{Q}^{1 / 2^{+}} \Longrightarrow \varepsilon_{a_{1} a_{2} a_{3}} Q_{a_{1}}\left(u_{a_{2}} C \gamma_{5} d_{a_{3}}\right), & \Lambda_{Q}^{1 / 2^{-}} \Longrightarrow \varepsilon_{a_{1} a_{2} a_{3}} \gamma_{5} Q_{a_{1}}\left(u_{a_{2}} C \gamma_{5} d_{a_{3}}\right) \\
\Lambda_{Q}^{3 / 2^{+}} \Longrightarrow \varepsilon_{a_{1} a_{2} a_{3}} \gamma_{5} Q_{a_{1}}\left(u_{a_{2}} C \gamma_{5} \gamma_{\mu} d_{a_{3}}\right), & \Lambda_{Q}^{3 / 2^{-}} \Longrightarrow \varepsilon_{a_{1} a_{2} a_{3}} Q_{a_{1}}\left(u_{a_{2}} C \gamma_{5} \gamma_{\mu} d_{a_{3}}\right) .
\end{array}
$$

The nonlocal generalizations of the above currents are used in the CCQM to evaluate the appropriate form factors and helicity amplitudes.

The numerical values of the normalized helicity amplitudes are listed in Table 1 . The helicity amplitudes $H_{\lambda_{2}, \lambda_{V}}$ of the produced $\Lambda^{(*)}$ states are clearly dominated by the helicity configuration $\lambda_{2}=-1 / 2$ as in the quark level transition $b \rightarrow s$. For the spin $1 / 2$ states in the transition $1 / 2^{+} \rightarrow$ $1 / 2^{ \pm}$this implies that the two $\Lambda^{(*)}(1 / 2)$ states are almost purely left-handed. 
Table 1: Moduli squared of normalized helicity amplitudes.

\begin{tabular}{|c|cccc|}
\hline$\Lambda^{*}$ & 1116 & 1405 & 1890 & 1520 \\
\hline$J^{P}$ & $\frac{1}{2}^{+}$ & $\frac{1}{2}^{-}$ & $\frac{3}{2}^{+}$ & $\frac{3}{2}^{-}$ \\
\hline$\left|\hat{H}_{+\frac{3}{2}+1}\right|^{2}$ & 0 & 0 & $3.50 \times 10^{-4}$ & $0.84 \times 10^{-4}$ \\
$\left|\hat{H}_{+\frac{1}{2}+1}\right|^{2}$ & $2.34 \times 10^{-3}$ & $1.27 \times 10^{-2}$ & $3.19 \times 10^{-2}$ & $2.26 \times 10^{-2}$ \\
$\left|\hat{H}_{+\frac{1}{2} 0}\right|^{2}$ & $3.24 \times 10^{-4}$ & $5.19 \times 10^{-3}$ & $1.61 \times 10^{-3}$ & $1.82 \times 10^{-3}$ \\
$\left|\hat{H}_{-\frac{1}{2} 0}\right|^{2}$ & 0.53 & 0.51 & 0.51 & 0.54 \\
$\left|\hat{H}_{-\frac{1}{2}-1}\right|^{2}$ & 0.47 & 0.47 & 0.45 & 0.44 \\
$\left|\hat{H}_{-\frac{3}{2}-1}\right|^{2}$ & 0 & 0 & $3.34 \times 10^{-3}$ & $1.06 \times 10^{-3}$ \\
\hline
\end{tabular}

\section{References}

[1] J. Lees et al. (BABAR Collaboration), Phys. Rev. Lett. 109, 101802 (2012), arXiv:1205.5442.

[2] R. Aaij et al. (LHCb Collaboration), Phys. Rev. Lett. 115, 111803 (2015) [Phys. Rev. Lett. 115, 159901 (2015)], arXiv:1506.08614.

[3] M. Huschle et al. (Belle Collaboration), Phys. Rev. D 92, 072014 (2015), arXiv:1507.03233.

[4] Y. Sato et al. (Belle Collaboration), Phys. Rev. D 94, 072007 (2016), arXiv:1607.07923.

[5] A. Abdesselam et al. (Belle Collaboration), arXiv:1608.06391.

[6] H. Na et al. (HPQCD Collaboration), Phys. Rev. D 92, 054510 (2015) Erratum: [Phys. Rev. D 93, 119906 (2016)], arXiv:1505.03925.

[7] S. Fajfer, J. F. Kamenik, and I. Nisandzic, Phys. Rev. D 85, 094025 (2012), arXiv:1203.2654.

[8] M. A. Ivanov, J. G. Körner and C. T. Tran, Phys. Rev. D 94, no. 9, 094028 (2016) [arXiv:1607.02932 [hep-ph]].

[9] R. Aaij et al. (LHCb Collaboration), Phys. Lett. B 724, 27 (2013) [arXiv:1302.5578 [hep-ex]].

[10] T. Gutsche, M. A. Ivanov, J. G. Körner, V. E. Lyubovitskij and P. Santorelli, Phys. Rev. D 88, no. 11, 114018 (2013) [arXiv:1309.7879 [hep-ph]].

[11] T. Gutsche, M. A. Ivanov, J. G. Körner, V. E. Lyubovitskij, V. V. Lyubushkin and P. Santorelli, Phys. Rev. D 96, no. 1, 013003 (2017) [arXiv:1705.07299 [hep-ph]].

[12] M. A. Ivanov, J. G. Körner and C. T. Tran, Phys. Rev. D 92, no. 11, 114022 (2015) [arXiv:1508.02678 [hep-ph]].

[13] M. A. Ivanov, J. G. Körner and C. T. Tran, Phys. Rev. D 95, no. 3, 036021 (2017) [arXiv:1701.02937 [hep-ph]]. 